\title{
Characteristics of Single-Event Upsets in a Fabric Switch (AD8151)
}

\author{
Stephen Buchner, Member, IEEE, Martin A. Carts, Member, IEEE, Dale McMorrow, Hak Kim, Member, IEEE, \\ Paul W. Marshall, Member, IEEE, and Kenneth A. LaBel, Member, IEEE
}

\begin{abstract}
Two types of single-event effects-bit errors and single-event functional interrupts-were observed during heavy-ion testing of the AD8151 crosspoint switch. Bit errors occurred in bursts with the average number of bits in a burst being dependent on both the ion linear energy transfer (LET) and on the data rate. A pulsed laser was used to identify the locations on the chip where the bit errors and single-event functional interrupts occurred. Bit errors originated in the switches, drivers, and output buffers. Single-event functional interrupts originated in the second-rank latch containing the data specifying the state of each switch in the $33 \times 17$ matrix.
\end{abstract}

Index Terms-Bit errors, fabric switch, heavy ions, pulsed laser, single-event functional interrupt.

\section{INTRODUCTION}

$\mathbf{F}$ ABRIC-BASED switches, such as crosspoint or crossbar switches, are finding widespread use in advanced communication networks, including storage area networks (SAN), synchronous optical networks (SONET), and Ethernets. These switches are capable of connecting multiple nodes together to significantly expand a network's effective data transmission rate. For example, fabric switches make possible the simultaneous transfer of data from multiple storage devices to multiple servers in SAN, leaving the main network free for other communications. For space applications, the ever-increasing demand for gathering and processing data on board spacecraft requires the use of a high-speed network to connect instruments to data processors and downlinks. Common data busses are incapable of handling large amounts of data due to their limited bandwidth and to the fact that access is restricted to one node at a time. Connecting several instruments together via a network can be accomplished with the use of fabric switches, such as the AD8151 Crosspoint switch.

In this paper, we present the first detailed investigation of single-event effects (SEEs) in a fabric-based switch exposed to

Manuscript received March 6, 2004; revised May 17, 2004. This work was supported by NASA Electronic Parts \& Packaging Program.

S. Buchner is with QSS Group, Inc., Seabrook, MD 20706 USA (e-mail: sbuchner@pop500.gsfc.nasa.gov).

M. A. Carts is with Raytheon Systems Corporation, Lanham, MD 20706 USA (e-mail: mcarts@pop500.gsfc.nasa.gov).

D. McMorrow is with Naval Research Laboratory, Washington, DC 20375

USA (e-mail: mcmorrow@ccs.nrl.navy.mil).

H. Kim is with Jackson \& Tull Chartered Engineers, Washington, DC 20018

USA (e-mail: hkim@pop700.gsfc.nasa.gov).

P. W. Marshall is an independent consultant to NASA GSFC, Brookneal, VA 24528 USA (e-mail: pwmarshall@aol.com).

K. A. LaBel is with NASA GSFC, Greenbelt, MD 20771 USA (e-mail: Kenneth.A.LaBel@nasa.gov).

Digital Object Identifier 10.1109/TNS.2004.835085 ionizing single particle (heavy ion) radiation. Our results reveal the presence of two distinct types of SEEs: single-event transients (SETs), also referred to as bit errors, and single-event functional interrupts (SEFIs). This classification has previously been applied to SEUs observed in other communication systems, including FireWire (IEEE 1394) and Myrinet Crossbar [1], [2]. We note that many of the bit errors take the form of bursts, where each burst may contain from one to more than 20 erroneous bits. Generally, bursts contain more erroneous bits for high-LET ions. We show how a pulsed laser was used to identify the spatial origins of the SEEs; the SEFIs originate in the registers containing the configuration data and in control lines, whereas the bit errors originate primarily in the switches themselves, but also in decoding circuits and output buffers.

\section{PART Description}

The Crosspoint switch selected for testing (AD8151) is capable of connecting 33 inputs to 17 outputs with a maximum data transfer rate of $3.2 \mathrm{~Gb} / \mathrm{s}$. The switch is nonblocking so that any available input can be connected to any available output, regardless of what other connections between inputs and outputs have previously been established.

Fig. 1 depicts the various functional blocks contained within the switch. Connecting a specific input to a specific output is accomplished by turning on the appropriate switch contained within the two-dimensional differential switch matrix. The state of a switch, whether "ON" or "OFF," is controlled by configuration data loaded sequentially into the first-rank latch and then strobed in parallel into the second-rank latch. The contents of the second-rank latch are decoded for driving the switches in the $33 \times 17$ matrix. The software provided by the manufacturer did not allow us to read the configuration data in the second-rank latch.

Although the actual circuit designs for the switch matrix and the individual switches in the AD8151 are proprietary, the design is fundamentally the same as shown in Fig. 2. That design will be used to illustrate the operation of the switch.

Fig. 2(a) is a diagram of a switch matrix with 33 inputs on the left and 17 outputs at the top. Any input can drive any output, provided that output is not already being used. It is possible to connect a single input to more than one output by turning on the requisite number of switches in the input's row. Therefore, the input amplifiers are designed with sufficient drive for 17 switches. Obviously, each output may be connected to only one input. At the top of each output column is a differential amplifier that senses the voltage difference between the two differential lines in each column. 


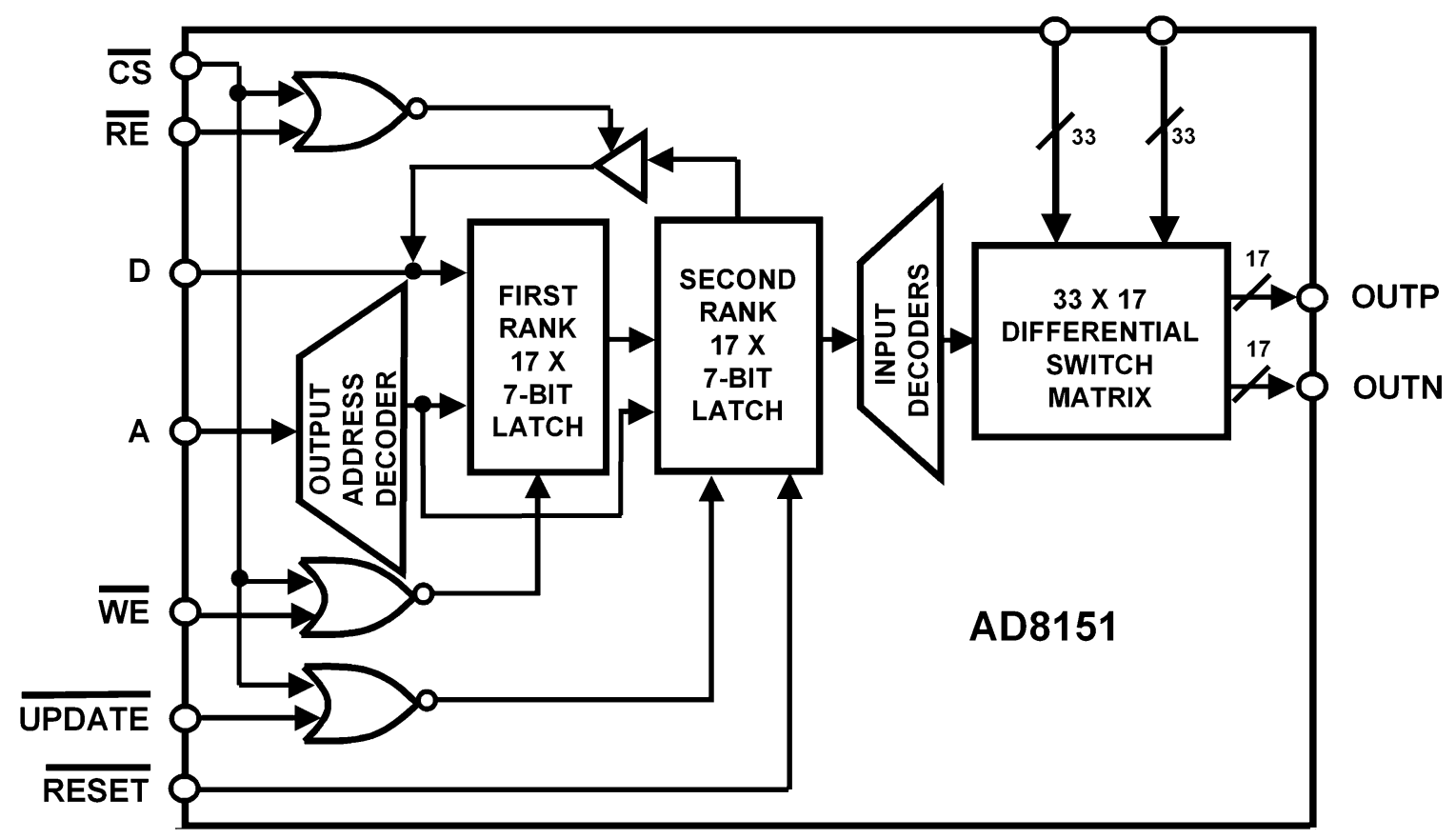

Fig. 1. Block diagram of the AD8151 Crosspoint Switch.

Fig. 2(b) is a circuit diagram of a simple bipolar switch. A decoder, controlled by data in the second rank latch, turns the switch on by connecting the emitters of the two bipolar transistors in the switch to a current source. Once the switch is turned on, a differential signal applied to the inputs propagates to the outputs. Conversely, when the switch is turned off the input signal is blocked from reaching the output.

Fig. 2 suggests that the parts of the switch matrix that may be sensitive to temporary upsets (bit errors) when exposed to ionizing particle radiation are the input drivers, output amplifiers and the switches themselves. On the other hand, SEUs in the second-rank latch containing configuration data for the switch will change the state of a switch and halt data transmission altogether.

\section{TEST SETUP}

To facilitate testing, the manufacturer provided a part mounted on an evaluation board together with software for controlling the switch. A bit-error-rate tester (BERT) was used for SEE testing. It supplied a serial stream of pseudorandom data to the switch input. The output of the switch was fed back to the BERT where it was compared with the original data pattern and any differences were flagged as bit errors. SETs consisted of either a single bit error or a burst of consecutive erroneous bits. These types of errors are classified as SETs because proper data transmission resumes following the event. The BERT signaled a SEFI when data transmission halted. Transmission could only be restored by reloading the configuration data into the second rank latch.

To determine how the error rate of the AD8151 in a radiation environment depended on the number of switches turned on, two different matrix configurations were tested. In the first configuration data was transmitted from one input to one output through one switch. In the second configuration five switches were turned on and outputs were connected to inputs in such a way as to allow data generated by the BERT to pass through the switch matrix five times before being read by the BERT. Fig. 3 shows the connections between inputs and outputs. Since the AD8151 is a nonblocking switch, any available input may be connected to any available output, regardless of whether the lines cross. Heavy-ion testing was done in air at Texas A\&M Cyclotron facility (TAMU) and pulsed laser testing at the Naval Research Laboratory (NRL). Measurements were taken for both switch configurations and for four different data transfer rates $(0.32,1,1.6$, and $3.2 \mathrm{~Gb} / \mathrm{s})$.

\section{RESULTS}

\section{A. Heavy-Ion Irradiation}

Two types of errors-SETs and SEFIs-were observed during heavy-ion testing of the AD8151.

SETs consisted of either a single bit error or a burst of errors. During a typical run involving ions with LETs $>5.72$ $\mathrm{MeV} \cdot \mathrm{cm}^{2} / \mathrm{mg}$, SETs were produced until a SEFI occurred, at which time data transmission ceased and the run was terminated. With increasing ion LET, SETs occurred more frequently and the associated error bursts contained more erroneous bits. However, SEFIs also occurred more frequently, which resulted in a reduction in the number of error bursts that could be detected before a SEFI stopped transmission.

When irradiated with ions having LETs $<5.72$ $\mathrm{MeV} \cdot \mathrm{cm}^{2} / \mathrm{mg}$, the part was insensitive to SEFIs and the SETs consisted of shorter bursts of errors. In those cases, runs were usually terminated after a preset fluence of $2 \times 10^{6} / \mathrm{cm}^{2}$ for which sufficient bit errors were recorded for meaningful statistics. 


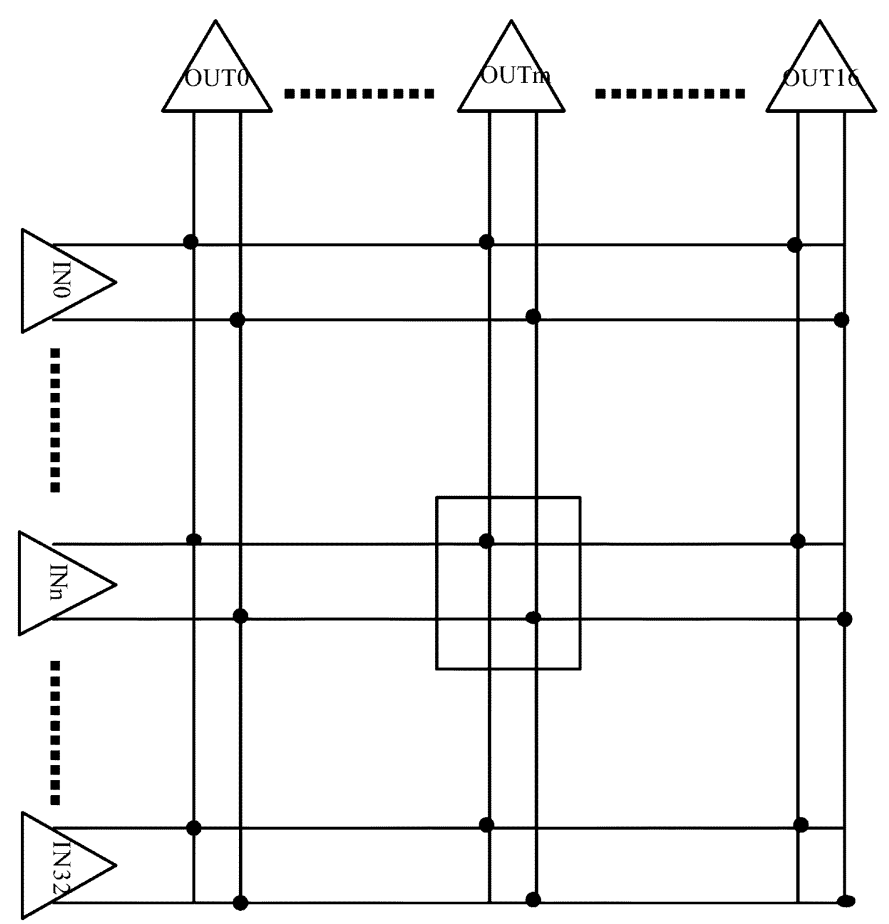

(2a)

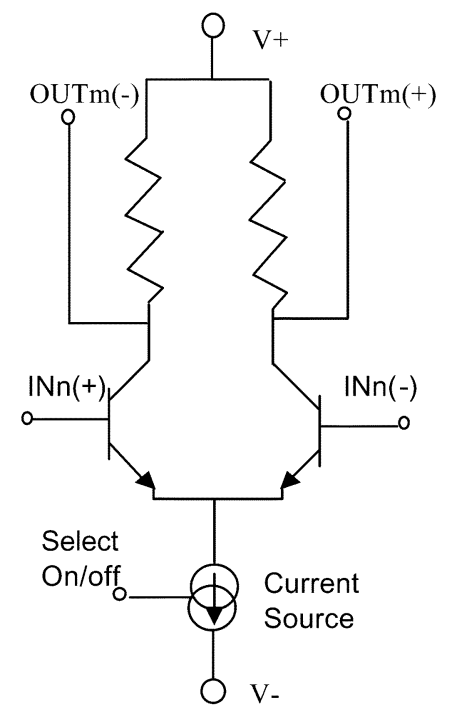

(2b)

Fig. 2. (a) Diagram showing connections for switch matrix. The switch in the box connects Input (n) to Output (m). (b) Circuit diagram of a typical bipolar switch connecting Input (n) to Output (m). The decoder determines which of the $33 \times 17$ switches in the matrix to turn on.

SET sensitivity was measured in terms of the event-error rate (EER), which is calculated using

$$
\mathrm{EER}=\mathrm{N}_{\mathrm{e}} /\left(\mathrm{N}_{\text {tot }} \cdot \Phi\right)
$$

where $\mathrm{N}_{\mathrm{e}}$ is the number of events, $\mathrm{N}_{\text {tot }}$ is the number of transmitted bits and $\Phi$ is the ion fluence. "Event" is defined as a series of errors of any length. Some of the events observed during testing consisted of a single erroneous bit, whereas others consisted of bursts containing more than 20 erroneous bits.

To illustrate how the data were analyzed, assume that 20 bits were transmitted before a SEFI halted transmission or the BERT

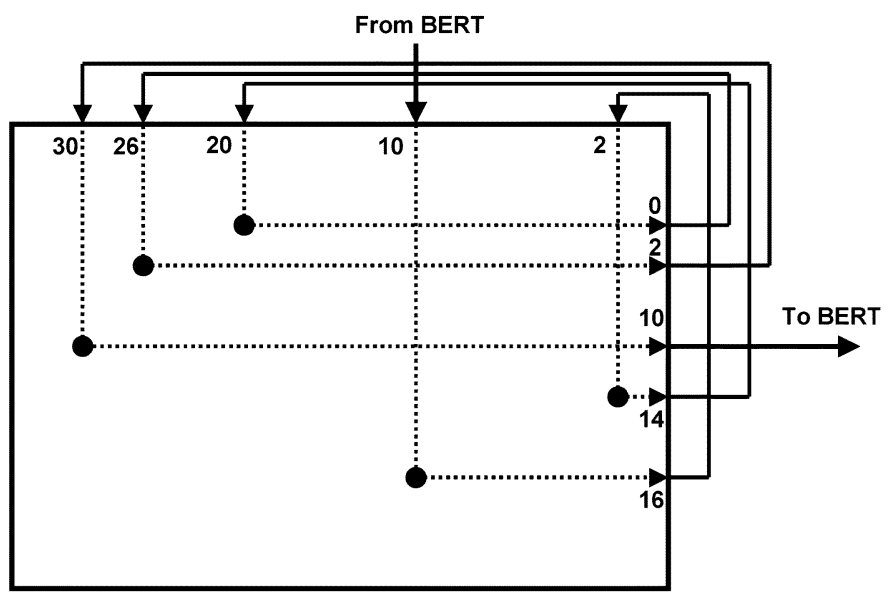

Fig. 3. Diagram illustrating the connections between five inputs and five outputs to form a continuous data transmission path through the AD8151 switch.

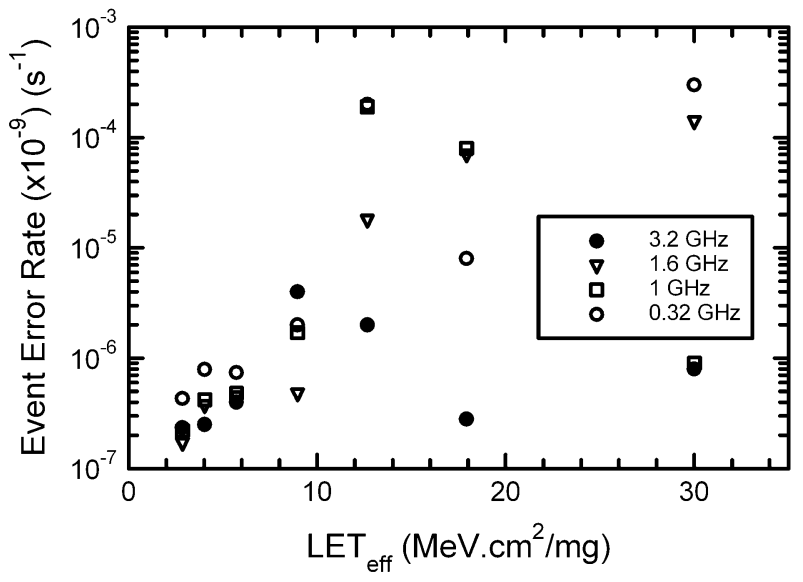

Fig. 4. Event error rate as a function of ion $\mathrm{LET}_{\text {eff }}\left(\mathrm{LET}_{\text {eff }}=\mathrm{LET} / \cos (\theta)\right.$ where $\theta$ is the ion's angle of incidence) for four different data transmission rates and five passes through the switch.

was turned off. The BERT determined that, of the 20 bits transmitted, the following were in error:

$$
\{1\}\{4,5,6\}\{8\}\{10,11\}\{16,17,18\}
$$

In this example, the number of transmitted bits $\left(\mathrm{N}_{\text {tot }}\right)$ was 20 , the number of events $\left(\mathrm{N}_{\mathrm{e}}\right)$ was 5 , the number of erroneous bits was 10, and the average number of bits in a burst was 2 .

Fig. 4 is a plot of the EER as a function of ion LET for four frequencies and five passes through the switch. Fig. 5 is a similar plot but for one pass through the switch. The graphs show that the LET threshold for SETs is below $2.86 \mathrm{MeV} \cdot \mathrm{cm}^{2} / \mathrm{mg}$. The EER increases with increasing LET and, for fixed LET, the EER generally decreases with increasing data rate. For a fixed fluence and flux, the total number of bits transmitted $\left(\mathrm{N}_{\text {tot }}\right)$ increases, which means the event error rate from (1) decreases. Also, the EER for five paths through the switch is higher by a factor of about five over that for a single path.

Fig. 6 shows the average burst length as a function of LET for four different frequencies and five passes through the switch. The data show that the average burst length increases with both LET and data transmission rate. 


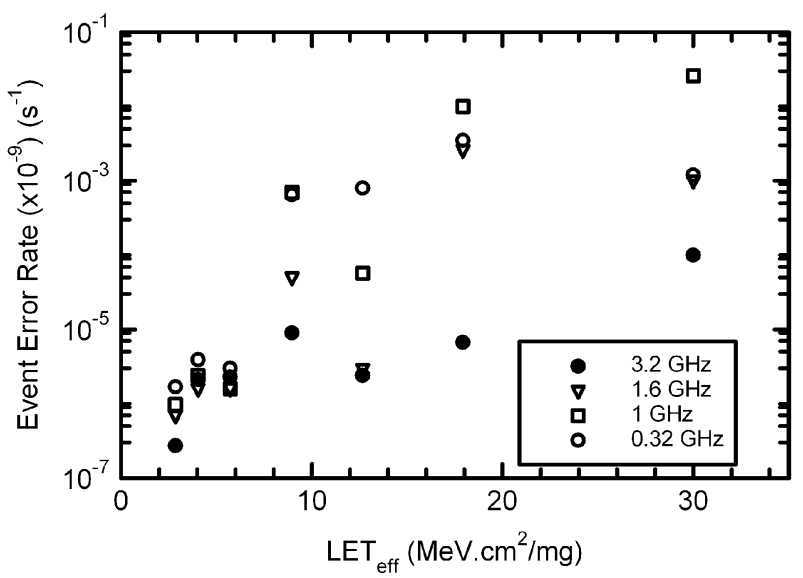

Fig. 5. Event error rate as a function of ion effective LET for four different transmission rates and one pass through the switch.

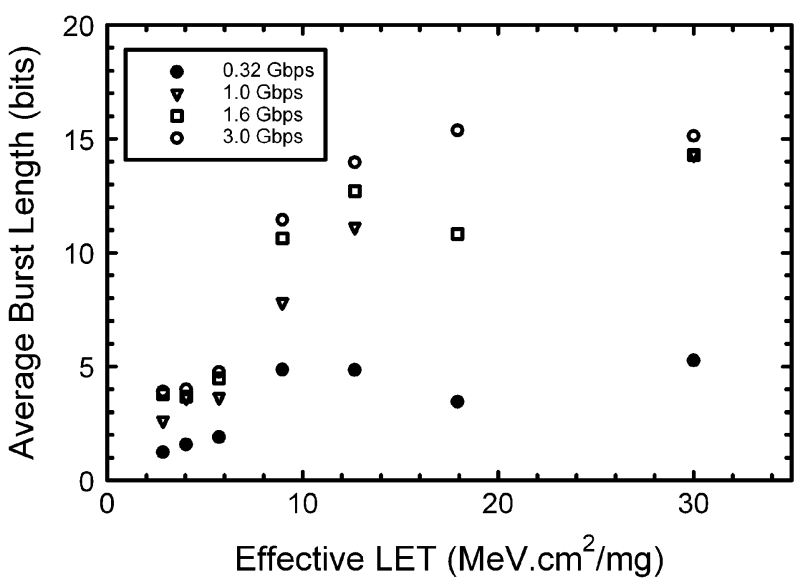

Fig. 6. Average length of error bursts as a function of ion effective LET for four different data rates and five paths through the switch.

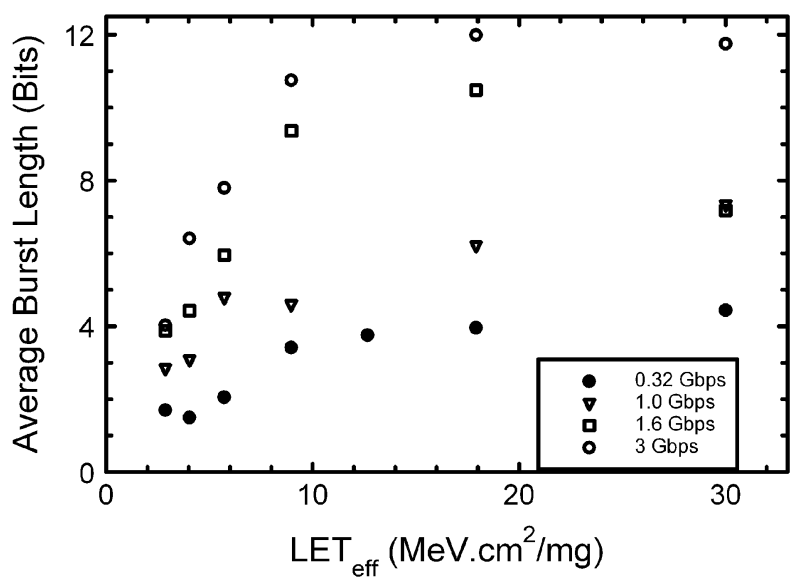

Fig. 7. Average length of error bursts as a function of ion effective LET for four different data rates and one path through the switch.

Fig. 8 is a plot of the SEFI cross-section $\left(\sigma_{\mathrm{SEFI}}\right)$ as a function of ion LET calculated by averaging the data for all transmission rates at each LET and based on the assumption that $\sigma_{\mathrm{SEFI}}$ was independent of data transmission rate. The data points are fit with a Weibull function whose parameters are listed in the graph's legend. The saturated SEFI cross-section for 5 paths through the switch was $2 \times 10^{-5} \mathrm{~cm}^{2}$ and the

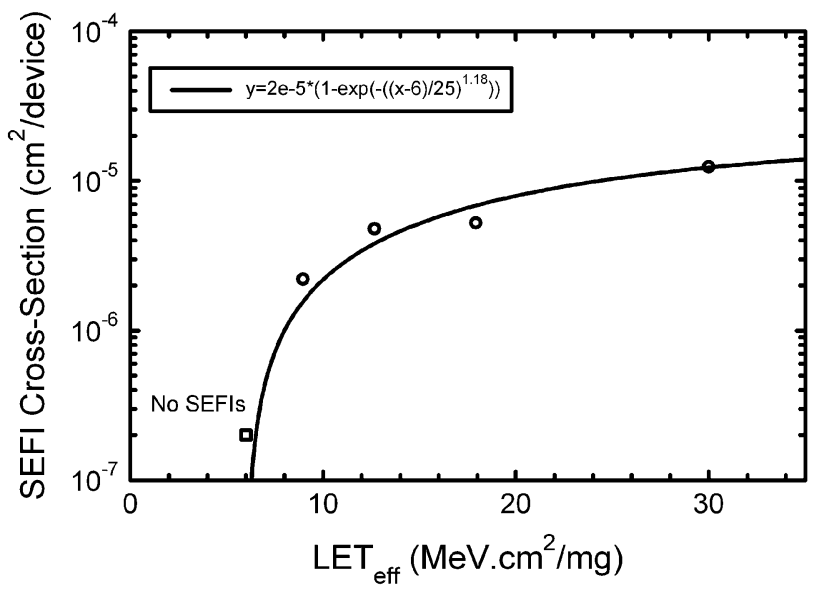

Fig. 8. SEFI cross section as a function of ion effective LET for four different data rates and five paths through the switch. The solid line is a fit to the data using a Weibull function.

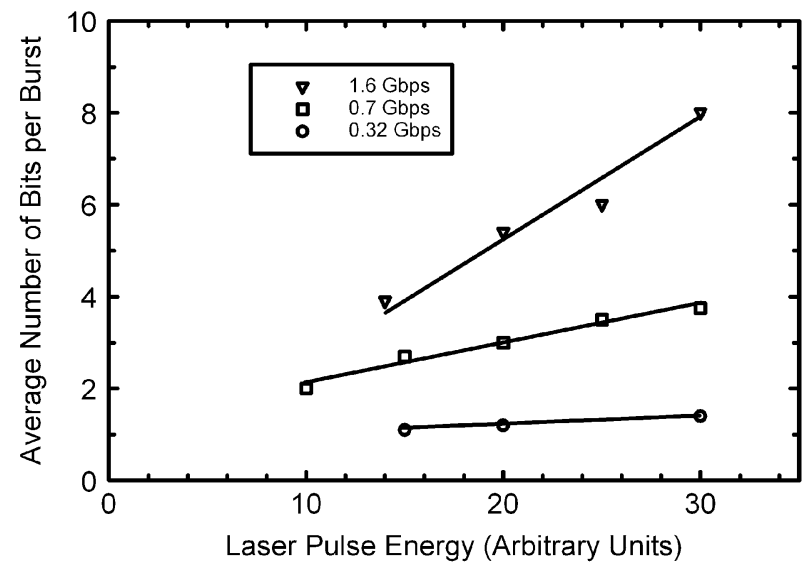

Fig. 9. Average number of bits in an error burst as a function of laser pulse energy for three different data transmission rates with the laser light focused on the switch. The data were fit with straight lines. The lowest point on the laser pulse energy scale at 10 A.U. is the threshold for producing SETs.

LET threshold was $6 \mathrm{MeV} \cdot \mathrm{cm}^{2} / \mathrm{mg}$. The SEFI cross section for one path through the switch had the same LET threshold $\left(6 \mathrm{MeV} \cdot \mathrm{cm}^{2} / \mathrm{mg}\right)$ as that for five paths, but the saturated cross-section $\left(3 \times 10^{-7} \mathrm{~cm}^{2}\right)$ was a factor of about 7 smaller, which is close to the expected ratio of 5 .

\section{B. Pulsed-Laser Irradiation Results}

A pulsed laser was used to probe all areas of the chip to identify the origins of SETs and SEFIs. Even though we were unable to obtain a detailed circuit diagram of the switch, we were able to distinguish the main functional blocks on the chip.

Bursts of errors were observed when switches in the seventh column (the output column) were irradiated with pulsed laser light. Of note is that every switch in the seventh column was SET sensitive and no switches in any of the other 16 columns were sensitive. Bursts of errors produced with pulsed laser light are similar to those observed when the part was irradiated with heavy ions.

The average burst length (ABL) was measured as a function of laser intensity and data rate at various locations on the chip. Fig. 9 shows the results obtained when the light was focused on 
switch \#1 in output column \#7. The data reveal that the ABL increases with both laser pulse energy and with data rate. For pulse energy of 30 (arbitrary units) the average burst length is about eight times longer for a data rate of $3.2 \mathrm{~Gb} / \mathrm{s}$ than for 0.32 $\mathrm{Gb} / \mathrm{s}$.

The light was scanned throughout the entire region where the decoding circuitry was located. Only the decoding circuitry associated with the seventh column was sensitive to SETs. This suggests that SETs in decoding circuitry affect only the switches in the active output column, which limits the sensitive area.

SETs were also generated when the light was focused on the output buffer at the end of the seven th column. Fig. 10 shows the $\mathrm{ABL}$ as a function of laser pulse energy and data rate. $\mathrm{ABL}$ scales linearly with laser pulse energy and increases with increasing data rate. Comparison of Figs. 9 and 10 reveals that the ABL of SETs generated in the buffer is longer than for SETs generated in the switches. Furthermore the energy threshold for generating SETs in the output buffer appears to be slightly higher because there is some data for the switch at energy 10 A.U.

The latches containing the configuration data (area D) were also scanned with the pulsed laser. The only kinds of SEUs observed were those causing a halt in data transmission. Without detailed information about the registers containing the configuration data, we were unable to draw any significant conclusions about which bits produced upsets.

\section{Discussion}

Temporary disruptions in data transmission are termed SETs. They are caused by ions passing near or through a transistor's sensitive junction. As they travel through the semiconductor material, the ions liberate electrons and holes that are subsequently separated by the junction's electric field. This process leads to a change in the voltage on the node that, if sufficiently large, can cause an off transistor to turn on. Eventually, the excess electrons and holes recombine, and the disturbed voltage returns to its original value. During the time the voltage is disturbed data transmission through a switch is interrupted.

The mechanism described above explains how a burst of errors occurs when an ion deposits charge in a switch. The "OFF" bipolar transistor in Fig. 2(b) may be turned "ON" if sufficient charge is deposited in its base-collector junction. The deposited charge causes the voltage on Out $(+)$ to drop and prevents the differential input voltage from reaching the output. This continues as long as the excess charge is present and all data bits transmitted during that time are lost. The lost bits together form a burst of consecutive errors. When all the excess charge has recombined, the switch resumes proper operation and the error burst comes to an end.

The dependence of the average burst length of an SET on ion LET (or laser pulse energy) is easily understandable in light of the above description. The amount of charge deposited by an ion or a pulse of laser light increases with ion LET or laser pulse energy, respectively. Because it takes longer for the additional charge to recombine, it follows that the duration of a SET increases with deposited charge, and the associate ABL also increases.

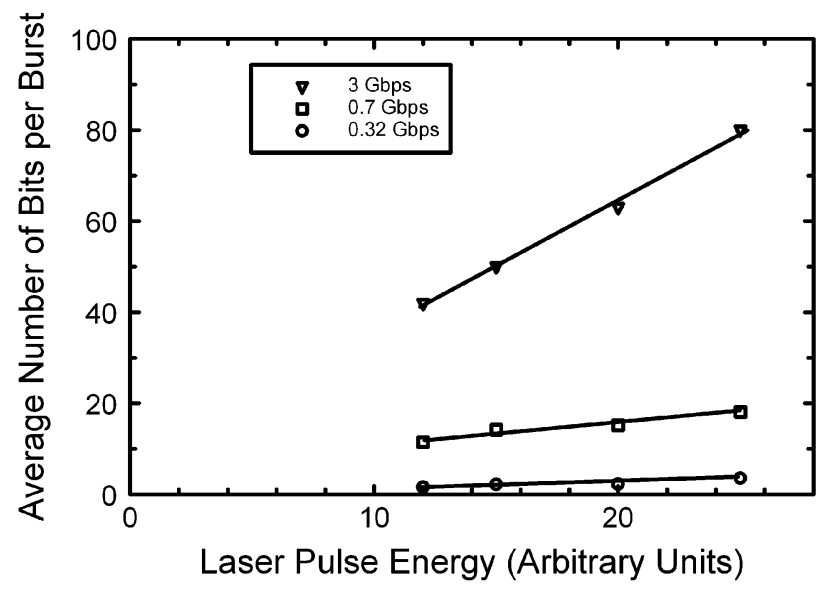

Fig. 10. Average number of bits in an error burst as a function of laser pulse energy for three different data transmission rates with the laser light focused on the output buffer in the seventh column. The data were fit with straight lines. The lowest point on the laser pulse energy scale is the threshold energy for producing SETs.

Figs. 6 and 7 show that the general trend is for the ABL to increase with ion LET, a dependence that is consistent with SETs having longer duration at higher LET. However, the increase is not monotonic with LET due to a number of possible reasons. The first is that the LET was varied in some cases by changing the angle of incidence, which might have an effect on the duration of the SET. The second is that ions also strike the configuration latch, producing SEFIs that stop transmission before sufficient bursts have been counted to obtain good statistical data.

Inspection of the raw data shows that there is a large distribution of burst lengths during each run, particularly for high LET ions. For example, for a LET of $30 \mathrm{MeV} \cdot \mathrm{cm}^{2} / \mathrm{mg}$, the length of a burst varies from a single bit to more than 100 bits, though the average is about 13 bits. Good statistical data is difficult to obtain at high LETs because SEFIs usually cause the switch to stop operating altogether before sufficient SETs have been collected.

The variations in burst length are caused by variations in ion strike location. First, more charge will be collected from a strike in the center of a sensitive node than a strike at the edge, leading to longer bursts for ion strikes in the most sensitive regions. This was demonstrated by moving the location of the focused laser light in the vicinity of a SET sensitive node and noting the length of the bursts. Second, different nodes have different sensitivities and a relatively insensitive node will produce shorter bursts than a more sensitive one.

Figs. 9 and 10 show that the ABL produced by pulsed laser light depends linearly on laser pulse energy. This is consistent with the time it takes for charge to be collection across a transistor junction - the more the amount of deposited charge, the longer it takes for the charge to be collected.

The same mechanisms of charge collection lead to the prediction that the ABL should be linearly dependent on data rate for a fixed ion LET. Simply stated, the faster the transmission rate, the more bits will be disturbed for a particular LET. Figs. 9 and 10 confirm that the ABL scales approximately linearly with data rate for fixed laser light energy and location. Fig. 9 shows, for example, that, for laser pulse energy of 30 arbitrary units, an 
increase in the data rate from $0.32 \mathrm{~Gb} / \mathrm{s}$ to $1.6 \mathrm{~Gb} / \mathrm{s}$ - a factor of 5-results in an increase in the ABL of from 1.5 to 8 error bits-a factor of 5.3. Although the ion data also show that the ABL increases with data rate for fixed ion LET, the increase from $0.32 \mathrm{~Gb} / \mathrm{s}$ to $3.2 \mathrm{~Gb} / \mathrm{s}$ is closer to a factor of 3 than the expected factor of 10 .

Figs. 4 and 5 show the EER as a function of ion LET for different data rates. Curves through to the data points have shapes very similar to those for cross section because, as can be seen in (1), the EER is just the cross section normalized by the bit transmission rate. In fact, a measure of the recovery time can be obtained from the data rate and the $\mathrm{ABL}$. Thus, for a data rate of $3 \mathrm{~Gb} / \mathrm{s}$ and a LET of $30 \mathrm{MeV} \cdot \mathrm{cm}^{2} / \mathrm{mg}$, the ABL was 14 bits, equivalent to a recovery time of $4.6 \mathrm{~ns}$.

A laser scan of the switch matrix for the case where input \#19 was connected to output \#7 revealed that the switch at the intersection of row \#19 and column \#7 was SEU sensitive, as were the other 32 switches in output column \#7. No switch in any other column was SET sensitive. This can be explained by referring to Fig. 2-charge deposited in the base/collector junction of the off transistor causes a drop in the output voltage that will be sensed by the output buffer and cause transmission to stop. Charge deposited in any other switch in column \#7 will also be sensed by the output buffer and result in a SET. Ion strikes to switches in other columns will cause the same effect on their outputs, but in the particular configuration tested here, those outputs are not connected, so no data passes through them. The input amplifiers are able to drive current through all switches in their rows. As a result, charge deposited by an ion in any switch in row \#19 other than the switch that is also in column \#7 will have no effect on data transmission from input \#19 to output \#7.

This finding has important implications for using this device in a radiation environment. One approach to hardening the device against SETs would be to minimize the path length through the switch matrix. For instance, connecting input \#1 to output \#1 results in the shortest path through the switch. However, because all 33 switches in the output column are SET sensitive, the SET rate should be no different from that obtained by connecting input row \#33 to output row \#17, the longest path through the switch. Furthermore, the SET rate should scale linearly with the number of connected outputs. This is in fact the case. The EER for five paths through the switch is roughly five times greater than for one path through the switch. We note that SETs generated in the output buffer and decoding logic make relatively small contributions to the total SET rate because their SET sensitive areas are small compared with the contributions from the 33 switches in each column. Furthermore, they have slightly higher LET thresholds than the switches.

At LETs greater than $6 \mathrm{MeV} \cdot \mathrm{cm}^{2} / \mathrm{mg}$, the SEU rate is dominated by more pernicious SEFIs, which cause a loss of transmission until the configuration data are reloaded into the second rank latch. The severity of the problem depends on the number of connections made in the switch. For a single connection only the data needed to specify the connection between input \#19 and output \#7 is critical. SEUs in that data will cause a SEFI. SEUs in data specifying the states of other switches will have no effect on data transmission. Clearly, as more connections are made between inputs and outputs, the amount of critical data in the second rank latch increases.

\section{SUMMARY AND CONCLUSION}

With the aid of the pulsed laser we were able to identify the origins of the SEUs in the AD8151 crossbar switch. Bit-errors occur when ions strike the decoding circuit, the switches, the output amplifier and the buffer. They are SETs because the nodes are only temporarily disturbed by the deposited charge and they have a recovery time that can be calculated from the data rate and the burst length. The relatively long bursts mean that simple error-detection-and-correction codes will not work in a radiation environment containing energetic heavy ions. We also found that all the switches in the output column are SEU sensitive regardless of which input is connected to that column. As a result, minimizing the path length through the switch cannot reduce the bit error rate. The results of the laser testing confirm that the EER should be greater for the configuration where the data passes five times through the switch as compared to where it passes only once through the switch. For five passes through the device, all the switches, amplifiers, buffers and decoders in five columns will be sensitive as compared to only one column for a single passage through the switch.

SEFIs occur when ions strike the registers in the second rank latch containing the configuration data. The heavy ion data show that a loss of transmission takes place after a smaller fluence for five paths through the switch than for one. The nonblocking design means that transmission will only be halted if a switch is turned off and for the case of five paths through the switch, there are five times as many switches to reprogram. Since SEFIs are generally more serious than bursts of bit errors, hardening the configuration registers to SEUs would greatly improve the reliability of the crossbar switch and can be done without compromising the high-speed performance of the device.

\section{REFERENCES}

[1] C. Seidleck, S. Buchner, H. Kim, P. Marshall, and K. LaBel, "Test methodology for characterizing the SEE response of a commerical IEEE 1394 serial bus (FireWire)," IEEE Trans. Nucl. Sci., vol. 49, pp. 3129-3134, Dec. 2002.

[2] J. Howard, K. LaBel, M. Carts, R. Stattel, C. Rogers, and T. Irwin, "proton single event effects (SEE) testing of the myrinet crossbar switch and network interface card," in Proc. 2002 IEEE Radiation Effects Data Workshop, Dec. 2002, pp. 32-40. 